OPEN ACCESS

Edited by:

Alessandra Durazzo, Council for Agricultural Research and

Economics, Italy

Reviewed by:

Iwona Konopka,

University of Warmia and Mazury in

Olsztyn, Poland

Biljana Rabrenović,

University of Belgrade, Serbia

Zeynep Aksoylu Özbek,

Celal Bayar University, Turkey

*Correspondence:

Wei-Ju Lee

weijulee@tmu.edu.tw

Specialty section

This article was submitted to

Nutrition and Food Science

Technology,

a section of the journal

Frontiers in Nutrition

Received: 24 August 2021 Accepted: 27 September 2021

Published: 21 October 2021

Citation

Tsao C-H, Chang C-W, Ho Y-C,

Chuang Y-K and Lee W-J (2021) Application of OXITEST for Prediction

of Shelf-Lives of Selected Cold-Pressed Oils.

Front. Nutr. 8:763524.

doi: 10.3389/fnut.2021.763524

\section{Application of OXITEST for Prediction of Shelf-Lives of Selected Cold-Pressed Oils}

\author{
Chieh-Hsi Tsao ${ }^{1}$, Chih-Wei Chang ${ }^{1}$, Yu-Chi Ho ${ }^{2}$, Yung-Kun Chuang ${ }^{3}$ and Wei-Ju Lee ${ }^{1,4 *}$ \\ ${ }^{1}$ School of Food Safety, Taipei Medical University, Taipei, Taiwan, ${ }^{2}$ Crop Improvement Section, Taoyuan District Agricultural \\ Research and Extension Station, Council of Agriculture, Executive Yuan, Taoyuan, Taiwan, ${ }^{3}$ Master Program in Food Safety, \\ Taipei Medical University, Taipei, Taiwan, ${ }^{4}$ Research Center of Food Safety Inspection and Function Development, College of \\ Nutrition, Taipei Medical University, Taipei, Taiwan
}

Introduction: Due to the enhanced awareness of consumers concerning healthy foods, homemade expeller-pressed oils have become popular worldwide. However, an extended storage period may lead to oxidization of the oil and exposure to hazardous byproducts by consumers.

Methods: In this study, 10 pressed oil samples prepared from common oilseeds using a small-scale expeller oil press were analyzed by OXITEST with a sample amount of $5 \mathrm{~g}$ of oil and an oxygen pressure of $800 \mathrm{kPa}$ under accelerated conditions for shelf-life projections. The oil properties were investigated, including the recovery, smoke point, acid value, iodine value, "fatty acid composition, and contents of pigments and tocopherols".

Results: The autoxidation reaction of various expeller-pressed oils under an accelerated testing system followed zero-order Arrhenius kinetics $\left(R^{2}>0.99\right)$. Shelf-lives of the pressed oils at $25^{\circ} \mathrm{C}$ were estimated by extrapolation to range $105 \sim 1,089$ days. The obtained shelf-lives were significantly correlated with log induction period (IP) values $(r>0.81, p<0.05)$ and unsaturated fatty acids (UFAs) $(r<-0.69, p<0.05)$, but not with the iodine value, acid value, or smoke point. Scatter diagrams between shelf-lives and UFAs suggested that these pressed oils could be grouped by two linear regression curves $(r>0.98, p<0.05)$. The predictive equations using multiple linear regression are presented herein, with predictor variables of UFAs and an unspecified item involving potential influencing factors such as tocopherol contents $(r>0.88, p<0.05)$.

Conclusions: Our findings first revealed that the UFA portion was partially correlated with the shelf-lives of selected expeller-pressed seed oils as estimated by the OXITEST. The derived equations can be applied for shelf-life predictions of expeller-pressed oils stored under dark ambient conditions based on the fatty acid profile.

Keywords: OXITEST, oxidative stability, shelf-life, expeller-pressed oil, induction period, unsaturated fatty acid, multiple regression model 


\section{INTRODUCTION}

Edible oils and fats are often acquired by mechanical pressing or solvent extraction followed by a refining process. Nowadays, the awakening of health consciousness in the general public has fostered the increasing popularity of mechanically pressed oils. Generally, pressed oils are processed without involving organic solvents and under a low temperature, possessing advantages of a low production of heat-induced chemical contaminants (13 ), preservation of more-beneficial minor components (4), and protection of unique flavors and odors for wide applications (5). Cold-pressed oils may be clarified by washing with water, settling, filtering, and centrifuging (6); however, they do not undergo a refining process of purification to eliminate minor compounds that are considered impurities and can potentially destabilize the oil (e.g., free fatty acids) or enhance oxidative stability (e.g., tocopherols) (7). Thus, safety concerns may arise from unintentionally consuming oxidized pressed oil, since it is not properly labeled and its shelf-life has not been evaluated. A suitable approach for estimating expeller-pressed oil shelf-lives is of importance to consumers to protect against ingesting oxidized oils and fats.

Unlike refined oils whose oxidative stability is mainly influenced by the fatty acid composition (8), the oxidative stability of pressed oils can be affected by several factors, including the fatty acid composition (9), natural antioxidants (10-12), prooxidants (13), and storage conditions (14). The existence of a correlation between tocopherols and fatty acids in vegetable oils has been discussed but still considered non-causal (15). Though the type of fatty acids is critically important for the oxidative stability of edible oils, substantial deviations were observed from the expectation that the higher the content of unsaturated fatty acids the lower the corresponding oxidative stability (6). Nevertheless, the negative trend of correlations between degree of unsaturation and oxidative stability was found to be absolute in previous studies $(6,15)$. Furthermore, the relative contribution of natural compounds to oxidative stability has proved elusive in edible oils and fats (6). In some cases, the seed-roasting process can significantly improve the oxidative stability of pressed oils by increasing antioxidant levels $(11,12)$, while extra virgin olive oil (EVOO) is rich in polyphenols, which are heat-sensitive natural antioxidants (10). Prooxidants such as chlorophylls can impair the stability of pressed oils. Pistachio oil, for example, has extremely low photo-oxidative stability due to the high contents of chlorophylls (13). Based on Taş and Gökmen's (16) literature review on total phenolic contents of various unroasted seeds including almond, peanut, pecan, pine nut, and walnut, similar low levels were found in those nuts without thermal treatment. Since expeller-pressed seed oils which are not subjected to a roasting process are regarded as coldpressed oils, their oxidative stabilities are hypothesized to be mainly influenced by the fatty acid composition and endogenous antioxidants like tocopherols.

Oxidative stability is often expressed as an induction period (IP), which is the period of time before a dramatic increase in the rate of lipid oxidation begins (17). There are several methods for evaluating the oxidative stabilities of lipids. Generally, samples are subjected to accelerated and standardized oxidation tests, including but not limited to the Schaal oven test, oxygen bomb method, active oxygen method (AOM), oxidative stability index (OSI, also called the Rancimat method), and OXITEST, all of which apply high temperatures (18). Among them, the OXITEST method (AOCS Cd 12c-16) directly detects changes in oxygen pressure inside a chamber and automatically determines the IP which avoids possible operative errors (19). Moreover, since oil storage conditions (e.g., light-preventing packaging material and oxygen exposure) can significantly affect the shelf-life $(14,20,21)$, the OXITEST system provides an ideal environment where light and oxygen exposures are well-controlled.

Currently there is no available standardized procedure for evaluating the shelf-life of pressed oils. Different shelf-life prediction models have been proposed to simulate EVOO degradation because of existing standards established by the Codex Alimentarius (22). For other pressed oils, the peroxide value $(\mathrm{POV}), \mathrm{p}$-anisidine value ( $\mathrm{p}-\mathrm{AV})$, and sensory evaluation are occasionally used to monitor quality changes and determine IPs during accelerated storage tests $(23,24)$. The shelf-lives of edible oils used to be estimated by plotting the log IP vs. elevated temperatures with satisfactory $R^{2}$ values of regression curves and extrapolation to ambient temperature to obtain the shelf-life (2527). Previous studies attempted to anticipate the shelf-life based on the IPs of edible kernels obtained by OXITEST $(28,29)$. Further research is warranted to better characterize the OXITEST method in shelf-life estimation for a wide variety of oil types and its correlation with physicochemical properties.

This study aimed to explore the potential use of the OXITEST as a tool to predict the shelf-life of expeller-pressed oils. Commercially available oilseeds in Taiwan were extracted by a small-scale, home-use oil expeller to prepare pressed oil samples. The effects of the sample amount and oxygen pressure on OXITEST IPs and suitable temperature ranges were first evaluated. Then, the IPs of pressed oils were determined at 70 , 90 , and $100^{\circ} \mathrm{C}$ by the OXITEST method, and extrapolated to generate ambient shelf-lives. The shelf-life results were correlated with the log IP, smoke point, acid value, iodine value, and ratio of saturated fatty acids (SFAs), unsaturated fatty acids (UFAs), polyunsaturated fatty acids (PUFAs), and the SFAs/UFAs ratio to develop predictive models. These properties are commonly available on product labels of pressed oils.

\section{METHODS}

\section{Preparation of Oil Samples}

All seeds and nuts including almonds, black sesame seeds, white sesame seeds, camellia seeds, golden linseed, peanuts, pecans, pine seeds, pumpkin seeds, sunflower seeds, and walnuts were purchased from local retailers (Taipei, Taiwan). Seed was homogenized using a Moulinette ${ }^{\circledR}$ kitchen blender (Moulinex, France) for $4 \mathrm{~min}$. Oil was extracted with an SX-TB02 screw oil press (Oiling Company, New Taipei City, Taiwan). Prior to pressing, the outlet nozzle was preheated to $70^{\circ} \mathrm{C}$ for $8 \mathrm{~min}$. Pressed oils were prepared by an initial pressing of the seeds and then a re-pressing of the cake from the first press. The oils were collected in centrifuge tubes and centrifuged at 3,000 relative 
centrifugal force (RCF) for $10 \mathrm{~min}$. The supernatant was decanted to another centrifuge tube, filled with nitrogen, and stored at $-80^{\circ} \mathrm{C}$ in the dark until being used.

Solvent extraction was undertaken to evaluate the oil content of different seeds. Seeds were first ground into a powder and dried at $50^{\circ} \mathrm{C}$ for $12 \mathrm{~h}$. Then, $10 \mathrm{~g}$ of dry powder was combined with $80 \mathrm{~mL}$ of $\mathrm{n}$-hexane. The mixture was ultrasonicated for $30 \mathrm{~min}$ and then filtered through Whatman no. 1 filter paper with suction using a Buchner funnel. The filtrate was removed from the extract using a rotary evaporator at $40^{\circ} \mathrm{C}$. The resulting oil was weighed, and the oil recovery was calculated.

\section{The OXITEST Method}

The OXITEST apparatus (OXITEST, Velp ${ }^{\circledR}$ Scientifica, Usmate, Italy) was implemented according to AOCS method no. Cd 12c16 (30). An oil sample was loaded into a hermetically sealed titanium chamber with a thermostat. The OXITEST reactor subjected the oil samples to an accelerated oxidative process via heating at $50 \sim 110^{\circ} \mathrm{C}$ and exposure to an oxygen pressure of $600 \sim 800 \mathrm{kPa}$. The instrument was controlled by original software (OXISoftTM, Velp ${ }^{\circledR}$ ) that monitored the oxygen pressure. At the end of the test, the program automatically calculated the IP from the resultant oxidation curves by the two-tangent method, i.e., the time required to reach the starting point of oxidation, corresponding to a sudden change in the rate of oxygen consumption (19).

\section{Selection of Parameter Values}

An optimization step was implemented to find suitable settings for the sample amount, oxygen pressure, and temperature range. Golden linseed oil was oxidized at $70^{\circ} \mathrm{C}$, at oxygen pressures of 600 and $800 \mathrm{kPa}$, and sample quantities of 5, 7.5, and $10 \mathrm{~g}$. Then, the IPs of golden linseed oil and walnut oil were analyzed at $50 \sim 110^{\circ} \mathrm{C}$, with an oxygen pressure of $800 \mathrm{kPa}$ and a sample amount of $5 \mathrm{~g}$.

\section{Sample Measurement}

The IPs of different expeller-pressed seed oils were analyzed by the OXITEST method. Oil samples at $5 \mathrm{~g}$ were loaded in the chamber with an oxygen pressure of $800 \mathrm{kPa}$ at 70,90 , and $100^{\circ} \mathrm{C}$.

\section{Shelf-Life Estimation}

Shelf-lives of expeller-pressed oils were estimated using the obtained IP values from the OXITEST method at 70, 90, and $100^{\circ} \mathrm{C}$. The equations of zero oxidation kinetics were calculated using the following equation, $\log \mathrm{IP}=\log \mathrm{IP}_{0}-\mathrm{k}_{0}$ t for different temperature values, where $\log \mathrm{IP}_{0}$ represents the initial Log IP value, $\mathrm{t}$ represents the temperature, $\mathrm{k}_{0}$ is a constant.

\section{Oil Properties}

The smoke point and acid value were respectively determined using AOCS official methods no. Cc 9a-48 and Cd 3d-63 (30). Carotenoids and chlorophylls were respectively determined at 470 and $670 \mathrm{~nm}$ by spectrophotometer (TC-1800 MK II, Tokyo, Japan) with $5 \%$ of oil in $n$-hexane (w/v) $(31,32)$. The pigment contents were calculated according to Boujemaa et al. (32).

The fatty acid profiles were determined based on modified AOCS official method no. Ce 2-66 to prepare the fatty acid methyl esters (FAMEs) (30). Analysis was performed by use of gas chromatography (Agilent 7890B, Agilent, CA, USA) equipped with DB-Heavy WAX column $(30 \mathrm{~m} \times 0.25 \mathrm{~mm}$ i.d., $0.25 \mu \mathrm{m}$ film thickness, Agilent, CA, USA) and a flame ionization detector. The flow rates for hydrogen and air were 30 and $300 \mathrm{~mL} / \mathrm{min}$, respectively. Hydrogen was used as a carrier gas with a flow rate at $4 \mathrm{~mL} / \mathrm{min}$. Nitrogen was used as makeup gas at a flow rate of $30 \mathrm{~mL} / \mathrm{min}$. The oven temperature was kept at $50^{\circ} \mathrm{C}$ for $2 \mathrm{~min}$, then increased to $170^{\circ} \mathrm{C}$ at a rate of $24^{\circ} \mathrm{C} / \mathrm{min}$, held for $5 \mathrm{~min}$, increased to $210^{\circ} \mathrm{C}$ at a rate of $3^{\circ} \mathrm{C} / \mathrm{min}$, followed by $230^{\circ} \mathrm{C}$ at a rate of $10^{\circ} \mathrm{C} / \mathrm{min}$ (held for $10 \mathrm{~min}$ ). The injection volume was $0.1 \mu \mathrm{L}$ and the mode was splitless direct injection. The detector temperature was $235^{\circ} \mathrm{C}$. The iodine value was directly calculated from the fatty acid composition according to AOCS official method no. Cd 1c-85 (30).

Tocopherol contents were analyzed by high-performance liquid chromatography-ultraviolet (HPLC-UV) based on AOCS official method no. Ce 8-89 with some modifications (30). In brief, HPLC system involved a Thermo ConstraMetric 3200 pump equipped with a Thermo SpectroMonitor $3200 \mathrm{UV} /$ vis detector. A reverse-phase analytical column YMC-Pack ODSAM $(250 \times 4.6 \mathrm{~mm}, 5 \mu \mathrm{m})($ YMC CO., LTD., Japan) was used for separation. The mobile phase was $2 \%(\mathrm{v} / \mathrm{v})$ methanol in ultrapure water (Milli-Q, Millipore) at a flow rate of $1.5 \mathrm{~mL} / \mathrm{min}$. The injection volume was $20 \mu \mathrm{L}$ and the detection wavelength was $292 \mathrm{~nm}$. Quantification was performed using external standard curves of $\alpha$ - and $\gamma$-tocopherol standards.

\section{Data Analysis}

Three biological replicates were performed for oil preparation. Then, all analyses were performed in triplicate $(n=3)$, except for fatty acid composition, tocopherol contents, pigments, and oxidative stability $(n=2)$. Data are presented as the mean $(n$ $=2)$ and mean \pm standard deviation (SD) $(n=3)$. Statistical analysis involved the use of Windows Excel 2019 (Microsoft, Redmond, WA, USA). Significant differences $(p<0.05)$ were determined by a two-tailed, paired Student's $t$-test. A simple linear regression analysis and multiple linear regression model were used to respectively determine the univariate and covariate associations. Pearson correlation coefficients were calculated, and the significance of associations was evaluated at the $p<0.05$ level.

\section{RESULTS}

\section{Oil Yields of Expeller-Pressed Oils}

In order to acquire freshly produced genuine pressed oils for an oxidative stability investigation, different oil seeds in Taiwan were collected and extracted in the laboratory using a commercial expeller oil press. As shown in Supplementary Table 1, the range of oil recoveries from different seeds prepared by the screw press was $22.8 \sim 62.5 \%$. Recoveries of most expeller-pressed oils were similar to those obtained by solvent extraction, except for camellia, peanut, and pine nut oils, which had significantly lower recoveries $(p<0.05)$ (Supplementary Table 1). Yields of pressed oils were calculated to be $56.4 \sim 104.5 \%$. In the present study, pressed oils were prepared by initial pressing of seeds and re-pressing of the cake of first press to achieve satisfactory 
TABLE 1 | Fatty acid compositions of 10 expeller-pressed oils.

\begin{tabular}{|c|c|c|c|c|c|c|c|c|c|c|c|c|c|c|}
\hline Oil type & C14:0 & C16:0 & C16:1 & C17:0 & C18:0 & C18:1 & C18:2 & C18:3 & C20:0 & C20:1 & C22:0 & SFA & UFA & PUFA \\
\hline Almond oil & 0.04 & 8.08 & 0.49 & 0.04 & 1.35 & 63.26 & 26.55 & 0.09 & 0.04 & 0.05 & ND & 9.55 & 90.44 & 26.64 \\
\hline Black sesame oil & 0.02 & 10.93 & 0.12 & 0.03 & 5.82 & 38.01 & 44.33 & ND & 0.55 & 0.12 & 0.08 & 17.43 & 82.58 & 44.33 \\
\hline Camellia oil & 0.04 & 10.49 & 0.12 & 0.04 & 1.82 & 78.30 & 8.79 & ND & ND & 0.39 & ND & 12.39 & 87.60 & 8.79 \\
\hline Golden linseed oil & 0.36 & 4.68 & ND & ND & 3.81 & 21.50 & 15.27 & 54.39 & ND & ND & ND & 8.85 & 91.16 & 69.66 \\
\hline Peanut oil & 0.02 & 7.41 & 0.03 & 0.09 & 3.45 & 79.12 & 4.91 & ND & 1.07 & ND & 2.39 & 14.43 & 84.06 & 4.91 \\
\hline Pecan oil & 0.06 & 8.15 & 0.07 & 0.05 & 2.37 & 57.95 & 31.11 & ND & 0.07 & 0.17 & ND & 10.70 & 89.30 & 31.11 \\
\hline Pine nut oil & ND & 6.22 & 0.08 & 0.04 & 2.59 & 28.04 & 45.13 & 16.09 & 0.35 & 1.39 & 0.07 & 9.27 & 90.73 & 61.22 \\
\hline Pumpkin seed oil & 0.11 & 13.63 & 0.11 & 0.09 & 6.07 & 31.98 & 47.31 & 0.27 & 0.36 & ND & 0.06 & 20.32 & 79.67 & 47.58 \\
\hline Sunflower seed oil & 0.06 & 6.75 & 0.09 & 0.03 & 3.38 & 36.61 & 51.97 & 0.2 & 0.18 & 0.12 & 0.60 & 11.00 & 88.99 & 52.17 \\
\hline Walnut oil & 0.35 & 6.04 & ND & ND & 2.52 & 14.76 & 60.00 & 16.34 & ND & ND & ND & 8.91 & 91.10 & 76.34 \\
\hline
\end{tabular}

Results are expressed as the mean $(n=2)$.

ND, not detectable. SFAs, saturated fatty acids; UFAs, unsaturated fatty acids; PUFAs, polyunsaturated fatty acids.

recoveries. Nevertheless, pine nuts and peanuts were more ductile due to their high moisture contents $(\sim 10 \%)$, resulting in less efficiency for oil separation from the seed residue and oil loss through the operation of the expeller press rod (31). On the contrary, low oil recovery of camellia seeds was probably due to the hardness of the seed. Camellia seeds are usually roasted prior to pressing to enhance oil extraction. Since the seed roasting process was proven to be a factor influencing camellia oil oxidative stability (33), it was excluded from our study design. These 10 selected seeds presented acceptable oil recoveries $(>20 \%)$ when pressed by the small-scale commercial screw oil press, indicating that all had potential to be used as raw materials for homemade expeller-pressed oils.

\section{Physicochemical Properties of Expeller-Pressed Oils}

The appearances of the expeller-pressed oils are presented in Supplementary Figure 1. Most of the oils were yellow at different saturations except for pumpkin seed oil. As shown in Supplementary Table 2, the values of carotenoids ranged from 0.51 to $10.08 \mathrm{mg} / \mathrm{kg}$, while the values of chlorophylls varied between 0.07 and $21.81 \mathrm{mg} / \mathrm{kg}$. Pumpkin seed oil appeared green and dark brown owing to the rich contents of chlorophylls and carotenoids, in agreement with Boujemaa et al. (32).

The fatty acid compositions of expeller-pressed oils are shown in Table 1. The predominant fatty acid was found to be oleic acid in almond oil (63.3\%), camellia oil (78.3\%), peanut oil $(79.1 \%)$, and pecan oil (58.0\%). The major fatty acids were linoleic acid and oleic acid in black sesame oil (44.3 and 38.0\%), pine nut oil (45.1 and 28.0\%), pumpkin seed oil (47.3 and $32.0 \%$ ), and sunflower seed oil (52.0 and 36.6\%), while linoleic acid, linolenic acid, and oleic acid were predominant in walnut oil. Golden linseed oil was abundant in PUFAs, particularly linolenic acid (54.4\%). The fatty acid compositions of expeller-pressed oils mentioned above paralleled previous studies (34-40). All of the theoretical iodine values of pressed oils were $<160 \mathrm{~g} \mathrm{I}_{2} / 100 \mathrm{~g}$ oil, except for that of linseed oil (Table 2). The iodine values of these pressed oils ranged from $76.6 \mathrm{~g} \mathrm{I}_{2} / 100 \mathrm{~g}$ for peanut oil to 187.2
TABLE 2 | Smoke points, acid values, and iodine values of 10 expeller-pressed oils.

\begin{tabular}{lccc}
\hline Oil type & $\begin{array}{c}\text { lodine value } \\
\left(\mathbf{g ~ I}_{\mathbf{2}} / \mathbf{1 0 0} \mathbf{g} \text { oil) }\right.\end{array}$ & Smoke point $\left.\mathbf{(}^{\mathbf{}} \mathbf{C} \mathbf{C}\right)$ & $\begin{array}{c}\text { Acid value } \\
\text { ( } \mathbf{m g ~ K O H} / \mathbf{g} \text { oil) }\end{array}$ \\
\hline Almond oil & 101.1 & $174.8 \pm 3.5$ & $0.53 \pm 0.07$ \\
Black sesame oil & 109.7 & $172.8 \pm 4.5$ & $3.89 \pm 0.08$ \\
Camellia oil & 83.0 & $176.5 \pm 3.5$ & $1.37 \pm 0.06$ \\
Golden linseed oil & 187.2 & $134.3 \pm 1.5$ & $1.70 \pm 0.07$ \\
Peanut oil & 76.6 & $141.8 \pm 2.8$ & $1.06 \pm 0.05$ \\
Pecan oil & 103.9 & $193.7 \pm 5.5$ & $0.23 \pm 0.01$ \\
Pine nut oil & 145.5 & $165.8 \pm 1.8$ & $1.51 \pm 0.05$ \\
Pumpkin seed oil & 110.3 & $184.3 \pm 4.2$ & $2.27 \pm 0.12$ \\
Sunflower seed oil & 122.2 & $109.0 \pm 5.1$ & $1.85 \pm 0.12$ \\
Walnut oil & 159.4 & $148.3 \pm 4.2$ & $2.26 \pm 0.07$
\end{tabular}

Results of the smoke point and acid value are expressed as the mean \pm standard deviation $(n=3)$, while iodine values are expressed as mean $(n=2)$.

${ }^{a}$ Calculated from the fatty acid compositions according to the AOCS Cd 1-85 method.

$\mathrm{I}_{2} / 100 \mathrm{~g}$ for linseed oil, reflecting a significant variation in the overall degree of saturation (Table 1 ).

The smoke points and acid values of the 10 pressed oils are shown in Table 2. Smoke points of the expeller-pressed oils ranged from 109.0 (sunflower seed oil) to $193.7^{\circ} \mathrm{C}$ (pecan oil). Unrefined oils tend to contain free fatty acids and other impurities, resulting in higher acid values and lower smoke points compared to refined oils (41). Acid values of the 10 seed oils were in a range of $0.20 \sim 3.89 \mathrm{mg} \mathrm{KOH} / \mathrm{g}$ oil, all within the specification of $<4 \mathrm{mg} \mathrm{KOH} / \mathrm{g}$ oil set by the Codex Alimentarius Commission (CAC) for cold-pressed and virgin oils (42).

\section{Optimization of the OXITEST Method}

The IPs obtained by the OXITEST with different weights of golden linseed oil and initial oxygen pressure settings at $70^{\circ} \mathrm{C}$ are shown in Table 3. The IPs of different weights (5, 7.5, and $10 \mathrm{~g}$ ) of golden linseed oil with $600 \mathrm{kPa}$ of oxygen pressure were in a range of $36.2 \sim 36.4 \mathrm{~h}$. The effect of the oil amount on the IP in the OXITEST system was considered negligible, consistent with 
TABLE 3 | Induction periods (IPS) of 5, 7.5, and $10 \mathrm{~g}$ of golden linseed oil determined by the OXITEST with oxygen pressures set to 600 and $800 \mathrm{kPa}$.

\begin{tabular}{lccc}
\hline Oxygen pressure & \multicolumn{3}{c}{ Oil weight } \\
\cline { 2 - 4 } & $\mathbf{1 0 . 0} \mathbf{~ g}$ & $\mathbf{7 . 5} \mathbf{~ g}$ & $\mathbf{5 . 0 ~ g}$ \\
\hline $600 \mathrm{kPa}$ & $36.3 \pm 0.4^{\mathrm{a}}$ & $36.4 \pm 0.2^{\mathrm{a}}$ & $36.2 \pm 0.2^{\mathrm{a}}$ \\
$800 \mathrm{kPa}$ & $33.8 \pm 0.1^{\mathrm{b}}$ & $34.5 \pm 0.0^{\mathrm{b}}$ & $34.6 \pm 0.1^{\mathrm{b}}$
\end{tabular}

Results are expressed as the mean \pm standard deviation $(n=3)$.

Different lowercase letters in the same column indicate a statistical difference $(p<0.05)$.

results of Comandini et al. (43) in that there were no significant differences among IPs using 3, 5, 7, or $10 \mathrm{~g}$ of sunflower oil at $110^{\circ} \mathrm{C}$. The sample amount was optimized to $5 \mathrm{~g}$ in order to minimize the amount of sample wasted while maintaining an even distribution of oil on the sample plate and regularizing the oxygen exposure. On the other hand, the obtained IPs with the oxygen pressure set to $800 \mathrm{kPa}$ were significantly shorter than those at $600 \mathrm{kPa}(p<0.05)$ (Table 3), indicating that the rate of lipid oxidation may increase under higher oxygen pressures. To reduce the required experimental time, the oxygen pressure parameter was adjusted to the upper limit of $800 \mathrm{kPa}$ in the following experiments.

The accelerated oxidation test was conducted in a temperature range of $50 \sim 110^{\circ} \mathrm{C}$ by the OXITEST reactor to investigate the linearity between log IP and temperature. The two pressed oils with the highest iodine values were tested. As shown in Supplementary Figure 2, linear correlations between log IP and temperature were good for walnut oil $\left(R^{2}=0.9992\right)$ and golden linseed oil $\left(R^{2}=0.9986\right)$. The IP of walnut oil at $110^{\circ} \mathrm{C}$ was $0.65 \mathrm{~h}$ (data not shown), which deviated from the regression curve and was therefore excluded. Similarly, Zhang et al. (44) analyzed the IP of Paeonia ludlowii kernel oil by the OXITEST at $70 \sim 110^{\circ} \mathrm{C}$ to extrapolate the shelf-life at room temperature. In that study, good linearity was found over a wide temperature range of $50 \sim 100^{\circ} \mathrm{C}$, suggesting that the OXITEST method depending on oxygen consumption could be time-saving for shelf-life estimations since the oxidation mechanism was not altered at high temperatures $\left(80 \sim 100^{\circ} \mathrm{C}\right)$.

\section{Oxidative Stability and Estimated Shelf-Life of Expeller-Pressed Oils}

IPs and estimated shelf-lives of 10 pressed oils assessed by the OXITEST method are given in Table 4. The IP decreased as the storage temperature increased, while the shelf-life was extended when the temperature decreased from 25 to $20^{\circ} \mathrm{C}$. A kinetic study was performed using OXITEST data to generate oxidation decay curves. As shown in Table 4, zero-order equations modeled the oxidation kinetics of the pressed oil samples at $70 \sim 100^{\circ} \mathrm{C}$ $\left(R^{2}>0.99\right)$. Shelf-lives of the pressed oils could therefore be predicted by extrapolation of the calibration curves at 70, 90, and $100^{\circ} \mathrm{C}$ owing to good linearity. The obtained rate constants $\left(\mathrm{k}_{0}\right.$ values) known as temperature acceleration factors were in the range of $0.033 \sim 0.043$ (Table 4). Although a poor correlation was found between rate constants and shelf-lives, a moderate positive correlation was observed between rate constants and iodine values $(r=0.67)$, indicating that the oxidation rate increased as the unsaturation degree was higher (data not shown). Peanut oil possessed the longest shelf-life with the presence of the highest $\log \mathrm{IP}_{0}$ compared to all other oils, followed by sesame oil, pecan oil, and pumpkin seed oil, which also showed higher $\log \mathrm{IP}_{0}$ values. Unlike rate constants, the obtained $\log \mathrm{IP}_{0}$ values were highly correlated with shelf-lives $(r=0.90)$.

In Table 4, shelf-lives at $25^{\circ} \mathrm{C}$ of expeller-pressed oils varied in a very wide range from $\sim 3$ months for almond oil to $\sim 3$ years for peanut oil at $25^{\circ} \mathrm{C}$. Also, differences in shelf-lives estimated at either 20 or $25^{\circ} \mathrm{C}$ are signified in Table 4 in consideration of seasonal or regional adjustment. The order of shelf-life was peanut oil $>$ black sesame oil $>$ pecan oil $\approx$ pumpkin seed oil $>$ pine nut oil $>$ camellia oil $>$ sunflower seed oil $\approx$ walnut oil $\approx$ golden linseed oil $>$ almond oil, in line with those of IP values at all temperatures. Since seed oils are rich in UFAs, greater oxidative stability was associated with a higher ratio of monounsaturated fatty acids (MUFAs) (45). Verardo et al. (45) investigated the oxidative stability of walnut samples, revealing an existing positive correlation between OXITEST results and the MUFA content $\left(R^{2}=0.8921, p<0.001\right)$ and a negative correlation between OXITEST results and the PUFA content $\left(R^{2}=-0.8865, p<0.001\right)$. The predominant fatty acid was oleic acid in peanut oil (79.12\%), pecan oil (57.95\%), and camellia oil (78.3\%), which exhibited relatively superior oxidative stabilities and longer shelf-lives (Table 4). Exceptionally, almond oil was the most susceptible to oxidation even though the fatty acid composition of was rich in oleic acid $(63.26 \%)$, which may be attributed to the contents and composition of tocopherols.

Tocopherol contents of pressed oils are presented in Supplementary Table 3. Tocopherols are antioxidants widely found in vegetable oils which can inhibit oxidation and therefore prolong the IP of those oils $(15,46)$. Generally, pressed seed oils are rich in tocopherols in either the $\alpha, \gamma$, or both forms, and the antioxidant efficacy of $\gamma$-tocopherol is known to be higher than that of $\alpha$-tocopherol $(15,46)$. Pecan oil, peanut oil, and golden linseed oil contained $\gamma$-tocopherol in appropriate high concentrations $(52.8 \sim 60.3 \mathrm{mg} / 100 \mathrm{~g}$ oil $)$, whereas lower $\gamma$-tocopherol levels were found in camellia oil, almond oil, and sunflower seed oil (10.1 16.5 mg/100 g oil). As a result, peanut oil and pecan oil were more oxidatively stable than almond, sunflower seed, and camellia oils during storage. Moreover, almond oil and sunflower seed oil possessed $\alpha$ tocopherol at high concentration ( $>30 \mathrm{mg} / 100 \mathrm{~g}$ oil) which may exhibit prooxidant effects $(15,46,47)$. As for golden linseed oil, low oxidative stability was mainly attributed to a high ratio of PUFAs (linolenic acid $>50 \%$ ) in the fatty acid composition. Similar to linseed oil, pine nut oil, walnut oil, and sunflower seed oil were primarily composed of PUFAs ( $>50 \%)$ and contained low to moderate levels of $\gamma$-tocopherol (16.5 36.3 mg/100 g oil), possessing relatively shorter induction periods. Sesame oil which was also rich in PUFAs and abundant in $\gamma$-tocopherol showed long shelf-lives, ranking second only to peanut oil. 
TABLE 4 | Induction periods (IPS), Arrhenius equations, and estimated shelf-lives of 10 expeller-pressed oils by the OXITEST method.

\begin{tabular}{|c|c|c|c|c|c|c|c|c|}
\hline \multirow[t]{2}{*}{ Oil type } & \multicolumn{3}{|c|}{ IP (h) } & \multicolumn{3}{|c|}{ Arrhenius equation ${ }^{a}$} & \multicolumn{2}{|c|}{ Estimated shelf-life (days) } \\
\hline & $70^{\circ} \mathrm{C}$ & $90^{\circ} \mathrm{C}$ & $100^{\circ} \mathrm{C}$ & $\log I_{0}$ & $\mathbf{k}_{0}$ & $\begin{array}{l}\text { Coefficient of determination } \\
\qquad\left(R^{2}\right)\end{array}$ & $20^{\circ} \mathrm{C}$ & $25^{\circ} \mathrm{C}$ \\
\hline Almond oil & 85.00 & 20.62 & 8.83 & 4.2126 & 0.0325 & 0.9979 & 152 & 105 \\
\hline Black sesame oil & 144.00 & 21.92 & 8.78 & 4.9952 & 0.0405 & 1.0000 & 638 & 400 \\
\hline Camellia oil & 85.22 & 14.20 & 6.63 & 4.5294 & 0.0372 & 0.9985 & 254 & 166 \\
\hline Golden linseed oil & 34.48 & 4.60 & 1.78 & 4.5457 & 0.0430 & 0.9998 & 202 & 123 \\
\hline Peanut oil & ND & 81.52 & 33.62 & 5.3799 & 0.0385 & 1.0000 & $1,697^{b}$ & $1,089^{b}$ \\
\hline Pecan oil & 151.00 & 25.08 & 10.11 & 4.9179 & 0.0391 & 1.0000 & 570 & 363 \\
\hline Pine nut oil & 74.00 & 10.88 & 4.32 & 4.7509 & 0.0412 & 0.9999 & 352 & 219 \\
\hline Pumpkin seed oil & 145.00 & 23.05 & 9.55 & 4.9211 & 0.0395 & 0.9999 & 564 & 358 \\
\hline Sunflower seed oil & 62.60 & 10.32 & 4.62 & 4.4458 & 0.0379 & 0.9992 & 203 & 131 \\
\hline Walnut oil & 44.47 & 6.50 & 2.67 & 4.5043 & 0.0409 & 0.9996 & 202 & 126 \\
\hline
\end{tabular}

Results are expressed as the mean $(n=2)$.

a Zero-order Arrhenius equations ( $\left.\log I P=\log I P_{0}-k_{0} t\right)$ are presented.

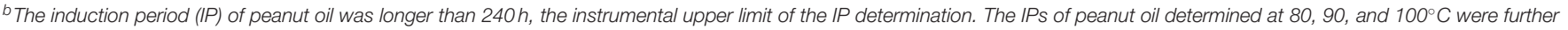
extrapolated to acquire the shelf-lives.

\section{Correlations Between Oil Properties and Shelf-Life}

Linear regression analyses were conducted between shelf-lives and $\log$ IPs, smoke points, acid values, and iodine values of expeller-pressed oils. Pumpkin seed oil was excluded because the endogenous chlorophyll could significantly influence the shelflife and cause a deviation from the other seed oils (32). As shown in Table 5, significant correlations were found between log IP values determined at different temperatures and shelf-lives $(p$ $<0.05$ ). However, it should be noted that since log IP values obtained by the OXITEST method were not totally parallel to shelf-lives, attention should be paid when comparing oxidative stability based on a single IP at one specific temperature. No significance was observed in the correlation analysis of the shelflife with the smoke point, acid value, or iodine value (Table 5). Although the initial acid value may considerably contribute to the oxidative stability at high temperature (25), our results demonstrated that the shelf-lives of pressed oils could not be predicted by either the smoke point, acid value, or iodine value. It is necessary to estimate the shelf-life based on a currently accepted qualified method.

As expected, the shelf-life was positively correlated with SFAs and the SFAs/UFAs ratio, while negatively correlated with UFAs and PUFAs (Table 5). A significant association was observed between UFAs and the shelf-life $(p<0.05)$, but not with SFAs, PUFAs, or the SFAs/UFAs ratio. Furthermore, these seed oils could be separated into two groups based on the linear regression results. Figure 1 presents the two-curve models, namely equations $\# 1$ and $\# 2$, between fatty acid compositions (SFAs, UFAs, SFAs/UFAs, and PUFAs) and shelf-lives at 20 and $25^{\circ} \mathrm{C}$. SFAs, UFAs, and SFAs/UFAs showed higher coefficients of determination $\left(R^{2}\right)$ compared to PUFAs, and UFAs exhibited the highest degree of correlation $\left(R^{2}>0.98\right)$ with shelf-lives at 20 and $25^{\circ} \mathrm{C}$. A multiple linear regression model approach was further adopted to simplify the equations, taking into account this new
TABLE 5 | Correlations of log induction period (IP) value, smoke point, acid value, "iodine value, and fatty acid compositions" with the shelf-life of nine expeller-pressed oils.

\begin{tabular}{|c|c|c|}
\hline & \multicolumn{2}{|c|}{ Correlation coefficient $(r)$} \\
\hline & Shelf-life at $20^{\circ} \mathrm{C}$ & Shelf-life at $25^{\circ} \mathrm{C}$ \\
\hline $\log \mathrm{IP}$ at $70^{\circ} \mathrm{C}$ & $0.9094^{\star}$ & $0.9124^{\star}$ \\
\hline $\log \mathrm{IP}$ at $90^{\circ} \mathrm{C}$ & $0.8394^{\star}$ & $0.8451^{\star}$ \\
\hline $\log I P$ at $100^{\circ} \mathrm{C}$ & $0.8177^{\star}$ & $0.8242^{*}$ \\
\hline Smoke point & -0.0197 & -0.0192 \\
\hline Acid value & -0.0890 & -0.0995 \\
\hline lodine value & -0.5222 & -0.5320 \\
\hline SFAs & 0.5910 & 0.5881 \\
\hline UFAs & $-0.6972^{*}$ & $-0.6951^{*}$ \\
\hline PUFAs & -0.5549 & -0.5654 \\
\hline SFAs/UFAs ratio & 0.6006 & 0.5975 \\
\hline
\end{tabular}

${ }^{*}$ Correlation was significant at the 0.05 level.

SFAs, saturated fatty acids; UFAs, unsaturated fatty acids; PUFAs, polyunsaturated fatty acids.

non-specific variable $\left(\mathrm{X}_{2}\right)$ along with fatty acid compositional ratios $\left(\mathrm{X}_{1}\right)$. As listed in Table 6, significant correlations were found for all regression curves between fatty acid ratios and shelflives at 20 and $25^{\circ} \mathrm{C}(p<0.05)$, except for PUFAs. The shelflife prediction model was established based on UFAs, because the correlation and significance were both stronger than for other variables (Figure 1). Moreover, the model was examined by testing the shelf-life of expeller-pressed white sesame seed oil. IPs of white sesame seed oil were determined to be 681 days at $25^{\circ} \mathrm{C}$ and 1,119 days at $20^{\circ} \mathrm{C}$ by the OXITEST method, which were similar to the predicted results of 692 days at $25^{\circ} \mathrm{C}$ and 1,084 days at $20^{\circ} \mathrm{C}$ based on UFAs, indicating that the prediction model was feasible and applicable. Expanding the applicability of this 


\section{A}

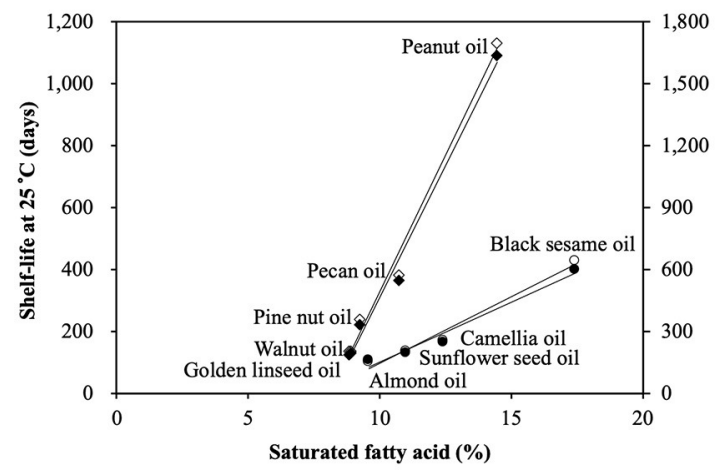

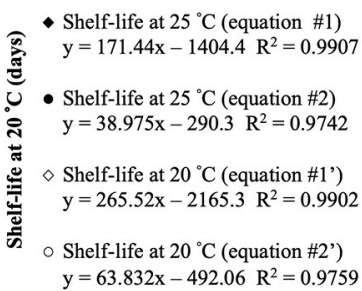

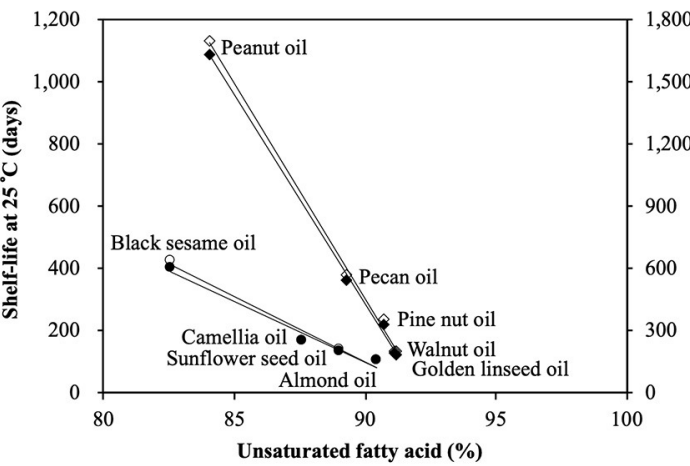

- Shelf-life at $25^{\circ} \mathrm{C}$ (equation \#1)

$\mathrm{y}=-134.82 \mathrm{x}+12419 \mathrm{R}^{2}=0.9978$

సิ $\mathrm{y}=-39.076 \mathrm{x}+3615.9 \mathrm{R}^{2}=0.9739$

气 $\diamond$ Shelf-life at $20^{\circ} \mathrm{C}$ (equation \#1')

$\mathrm{y}=-208.82 \mathrm{x}+19246 \mathrm{R}^{2}=0.9974$

क $\circ$ Shelf-life at $20^{\circ} \mathrm{C}$ (equation \#2')

$y=-63.998 x+5905.3 R^{2}=0.9756$

C

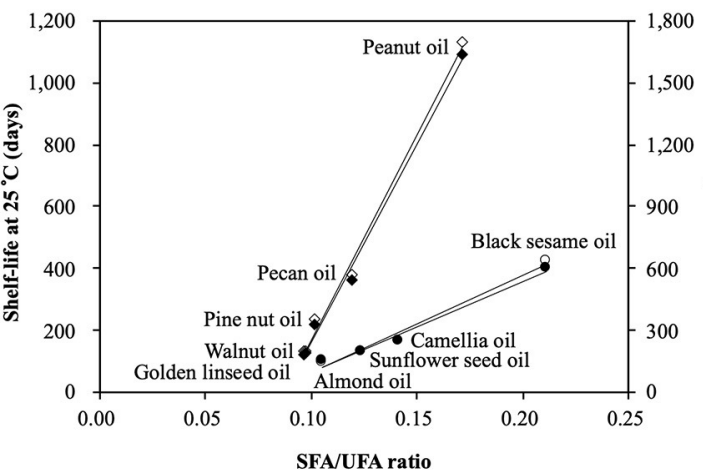

- Shelf-life at $25^{\circ} \mathrm{C}$ (equation \#1)

(a $y=12840 x-1127 \mathrm{R}^{2}=0.9944$

త్

- Shelf-life at $25^{\circ} \mathrm{C}$ (equation \#2)

$y=2910 x-223 R^{2}=0.9808$

$\diamond$ Shelf-life at $20^{\circ} \mathrm{C}$ (equation \#1')

$\mathrm{y}=19881 \mathrm{x}-1736 \mathrm{R}^{2}=0.9940$

ह Shelf-life at $20^{\circ} \mathrm{C}$ (equation \#2')

$\mathrm{y}=4757 \mathrm{x}-380 \mathrm{R}^{2}=0.9823$

D

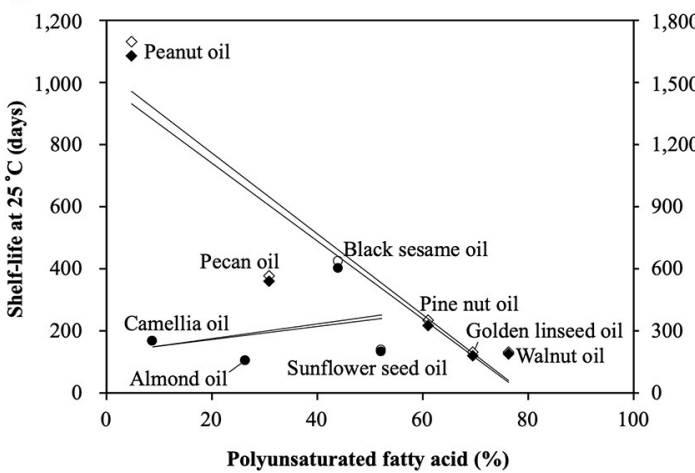

Shelf-life at $25^{\circ} \mathrm{C}$ (equation \#1) $y=-12.593 x+996.61 \quad R^{2}=0.8627$ है

- Shelf-life at $25^{\circ} \mathrm{C}$ (equation \#2) $y=2.1121 x+130.84 R^{2}=0.0911$

$\diamond$ Shelf-life at $20^{\circ} \mathrm{C}$ (equation \#1') $\mathrm{y}=-19.503 \mathrm{x}+1553.4 \mathrm{R}^{2}=0.862$

के Shelf-life at $20^{\circ} \mathrm{C}$ (equation \#2') $y=3.5867 x+193.45 R^{2}=0.0981$

FIGURE 1 | The two-curve models between fatty acid compositions and shelf-lives at 20 and $25^{\circ}$ C: (A) Saturated fatty acid (SFA); (B) Unsaturated fatty acid (UFA); (C) SFA/UFA ratio; (D) Polyunsaturated fatty acid (PUFA). 
TABLE 6 | Multiple regression analyses between the fatty acid composition and shelf-life of nine expeller-pressed oils.

\begin{tabular}{|c|c|c|c|c|c|c|}
\hline \multirow[t]{2}{*}{$x_{1}$} & \multicolumn{3}{|c|}{ Shelf-life at $20^{\circ} \mathrm{C}(\mathrm{Y})$} & \multicolumn{3}{|c|}{ Shelf-life at $25^{\circ} \mathrm{C}(\mathrm{Y})$} \\
\hline & Equation $^{a}$ & Correlation coefficient $(r)$ & $p$ & Equation $^{a}$ & Correlation coefficient $(r)$ & $p$ \\
\hline SFAs & $y=142 X_{1}+600 X_{2}-1,474$ & 0.8374 & 0.0266 & $y=90 x_{1}+378 x_{2}-935$ & 0.8291 & 0.0306 \\
\hline UFAs & $y=-138 X_{1}+550 X_{2}+12,337$ & 0.8953 & 0.0078 & $y=-88 X_{1}+347 X_{2}+7868$ & 0.8885 & 0.0093 \\
\hline SFAs/UFAs & $y=10563 X_{1}+586 X_{2}-1,224$ & 0.8376 & 0.0266 & $y=6717 X_{1}+370 X_{2}-776$ & 0.8292 & 0.0305 \\
\hline PUFAs & $y=-14 X_{1}+512 X_{2}+773$ & 0.7615 & 0.0741 & $y=-9 x_{1}+326 X_{2}+500$ & 0.7666 & 0.0701 \\
\hline
\end{tabular}

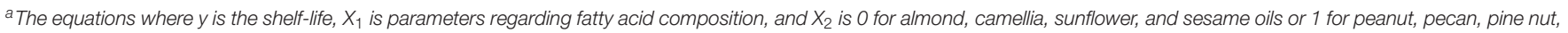
walnut, and golden linseed oils.

SFAs, saturated fatty acids; UFAs, unsaturated fatty acids; PUFAs, polyunsaturated fatty acids.

model to more types and species of oils requires further study in the future.

The unspecified variable $\left(\mathrm{X}_{2}\right)$ could be explained by the variation of tocopherol content and composition. CasteloBranco et al. (48) proposed using the antioxidant capacity as a surrogate measure for the stability of vegetable oils. It was also reported that $\gamma$-tocopherol was the most important predictor in the models (48). Since tocopherols are efficient antioxidants at low concentrations but they gradually lose efficacy as their concentrations in the vegetable oils increase, defeating the purpose of correlating with shelf-life $(31,46,47)$. In our study, the grouped pressed oils (i.e., linseed oil, walnut oil, peanut oil, and pecan oil fitted in equation \#1) showing better resistance to oxidation with an increasing UFA ratio tended to contain higher levels of $\gamma$-tocopherol than the other group (i.e., almond oil, camellia oil, and sunflower seed oil fitted in equation \#2) (Supplementary Table 3). Pine nut oil contained both $\gamma$-tocopherol and $\alpha$-tocopherol at similar and appropriate levels which are supposed to elevate the antioxidant activity. An exception occurred in that sesame oil principally contained $\gamma$-tocopherol but was grouped in equation \#2, which was likely due to the $\gamma$-tocopherol content being high enough (> $100 \mathrm{mg} / 100 \mathrm{~g}$ oil) to be prooxidant $(15,47)$. Since $\gamma$ tocopherol has a stronger antioxidant effect than $\alpha$-tocopherol, the slope values of equation \#1 appeared to be higher than those of equation $\# 2$.

Although correlations among tocopherols, polyphenols, and fatty acid compositions are still controversial $(6,15,48)$, our findings demonstrated that the unsaturated degree was the most crucial factor for these selected homemade expeller-pressed seed oils. The forms and levels of tocopherols also play important roles in predicting the shelf-life of these seeds, consistent with our hypothesis that the major influencing factors for shelf-lives of expeller-pressed seed oils are the UFA ratio and antioxidants, mainly tocopherols.

\section{DISCUSSION}

Cold-pressed oils are frequently chosen by consumers due to their reputation as a good source of natural nutrients and ingredients from seeds. According to previous studies, the oxidative stability of pressed oils can be affected by multiple factors, such as the degree of saturation of fatty acids, minor compound constituents, the processing temperature, and storage conditions $(8,11,14,33,49)$. In the present study, the oxidative stability of expeller-pressed oils was first studied using the OXITEST system to develop a prediction model in order to assess ambient storage shelf-lives. To achieve this aim, the oil type, processing conditions, and an oxidative stability evaluation method were restricted to decrease the impacts of confounding variables described below. First, the processes of drying and expeller-pressing for pressed oil preparation were consistent. Roasting effects were not addressed in this study, because thermal treatment may significantly enhance the oxidative stability of pressed oils $(12,33,49)$. Second, the IPs measured by OXITEST were less likely to be affected by light, oxygen, and anthropic factors, since it automatically monitors changes in the oxygen pressure in a sealed chamber with software controlling the entire operation and calculating the IP. In addition, effects of the sample amount and operation temperature that ranged up to $100^{\circ} \mathrm{C}$ on the resultant shelf-life were insignificant based on our results, while the IPs became significantly shorter as the oxygen pressure increased from 600 to $800 \mathrm{kPa}$. Therefore, shelf-life prediction from the extrapolation of Arrhenius plots should be only applied to freshly prepared pressed oils stored in an opaque bottle with as low oxygen exposure as possible. Last, minor constituents like tocopherols and polyphenols in the pressed oils may increase the complexity of shelf-life predictions. Pumpkin seed oil was excluded as a notable exception from the prediction equation due to the occurrence of chlorophylls. For the other nine seeds, this study first revealed an unspecified variable $\left(\mathrm{X}_{2}\right)$ parceled the latent impacts of other minor constituents mainly tocopherols in the final regression equation. Furthermore, the equations seemed to be applicable for predicting the shelflife of different varieties of these seeds (i.e., black and white sesame seeds).

Herein, ambient storage shelf-lives of freshly prepared expeller-pressed oils were estimated by the OXITEST, and a prediction model was first established based on an association with the fatty acid composition. A significant correlation was found between the shelf-life and UFA ratio, suggesting that the fatty acid composition is one of the crucial factors in pressed oil stability. The scatter diagrams appeared to fit the multiple linear regression model with two predictor variables, where $\mathrm{X}_{1}$ was the UFA ratio and $\mathrm{X}_{2}$ was defined as 0 or 
1 for different types of seeds $\left(R^{2}>0.98\right)$, which shed new light on prediction models of expeller-pressed oil shelf-life. Nonetheless, the current study is limited by the small sample size and restricted experimental conditions: roasting process, storage environment, and the compositional variances in oils such as polyphenol contents were not determined. Further research involving larger sample sizes should be done to elucidate other potential influencing factors.

\section{DATA AVAILABILITY STATEMENT}

The raw data supporting the conclusions of this article will be made available by the authors, without undue reservation.

\section{AUTHOR CONTRIBUTIONS}

C-HT: investigation and writing-original draft preparation. $\mathrm{C}-\mathrm{WC}$ : investigation. Y-CH: resources. Y-KC: methodology. W-JL: conceptualization, funding acquisition, supervision, and

\section{REFERENCES}

1. Zhao M, Lan Y, Cui L, Monono E, Rao J, Chen B. Formation, characterization, and potential food application of rice bran wax oleogels: expeller-pressed corn germ oil versus refined corn oil. Food Chem. (2020) 309:125704. doi: 10.1016/j.foodchem.2019.125704

2. Chen $\mathrm{H}$, Tsao $\mathrm{CH}$, Chang $\mathrm{YH}$, Lee WJ. Occurrence of thermally induced glycidyl esters and 3-monochloropropane-1,2-diol esters in refined oils and pressed oils manufactured by different processes and associated with human health risks in Taiwan. Food Chem. (2021) 360:130053. doi: 10.1016/j.foodchem.2021.130053

3. Ramadan MF. Cold Pressed Oils: Green Technology, Bioactive Compounds, Functionality, and Applications. Cambridge: Academic Press (2020). p. 774. doi: 10.1016/B978-0-12-818188-1.00001-3

4. Ghazani SM, Marangoni AG. Minor components in canola oil and effects of refining on these constituents: a review. J Am Oil Chem Soc. (2013) 90:923-32. doi: 10.1007/s11746-013-2254-8

5. Zhang W, Cao X, Liu SQ. Aroma modulation of vegetable oils-A review. Crit Rev Food Sci Nutr. (2020) 60:1538-51. doi: 10.1080/10408398.2019.1579703

6. Redondo-Cuevas L, Castellano G, Torrens F, Raikos V. Revealing the relationship between vegetable oil composition and oxidative stability: a multifactorial approach. J Food Compost Anal. (2018) 66:221-9. doi: 10.1016/j.jfca.2017.12.027

7. Febrianto NA, Yang TA. Producing high quality edible oil by using ecofriendly technology: a review. J Food Sci Technol. (2011) 3:317-26. Available online at: http://maxwellsci.com/print/ajfst/v3-317-326.pdf

8. Vidrih R, Vidakovič S, Abramovič H. Biochemical parameters and oxidative resistance to thermal treatment of refined and unrefined vegetable edible oils. Czech J Food Sci. (2010) 28:376-84. doi: 10.17221/202/2008-CJFS

9. Borges TH, Pereira JA, Cabrera-Vique C, Lara L, Oliveira AF, Seiquer I. Characterization of Arbequina virgin olive oils produced in different regions of Brazil and Spain: physicochemical properties, oxidative stability and fatty acid profile. Food Chem. (2017) 215:454-62. doi: 10.1016/j.foodchem.2016.07.162

10. Conte L, Milani A, Calligaris S, Rovellini P, Lucci P, Nicoli MC. Temperature dependence of oxidation kinetics of extra virgin olive oil (EVOO) and shelflife prediction. Foods. (2020) 9:295. doi: 10.3390/foods9030295

11. Vaidya B, Choe E. Effects of seed roasting on tocopherols, carotenoids, and oxidation in mustard seed oil during heating. J Am Oil Chem Soc. (2011) 88:83-90. doi: 10.1007/s11746-010-1656-0 writing-review and editing. All authors contributed to the article and approved the submitted version.

\section{FUNDING}

This work was supported by Taipei Medical University (project no. TMU106-AE1-B39) and the Taiwanese Ministry of Science and Technology (project no. MOST108-2813-C-038-028-B).

\section{ACKNOWLEDGMENTS}

The authors acknowledge the technical support provided by the Taipei Medical University Core Facility.

\section{SUPPLEMENTARY MATERIAL}

The Supplementary Material for this article can be found online at: https://www.frontiersin.org/articles/10.3389/fnut.2021. 763524/full\#supplementary-material

12. Lee SW, Jeung MK, Park MH, Lee SY, Lee J. Effects of roasting conditions of sesame seeds on the oxidative stability of pressed oil during thermal oxidation. Food Chem. (2010) 118:681-5. doi: 10.1016/j.foodchem.2009.05.040

13. Ling B, Yang X, Li R, Wang S. Physicochemical properties, volatile compounds, and oxidative stability of cold pressed kernel oils from raw and roasted pistachio (Pistacia vera L. Var Kerman). Eur J Lipid Sci Tech. (2016) 118:1368-79. doi: 10.1002/ejlt.201500336

14. Kanavouras A, Coutelieris F. Shelf-life predictions for packaged olive oil based on simulations. Food Chem. (2006) 96:4855. doi: 10.1016/j.foodchem.2005.01.055

15. Kamal-Eldin A. Effect of fatty acids and tocopherols on the oxidative stability of vegetable oils. Eur J Lipid Sci Tech. (2006) 108:105161. doi: 10.1002/ejlt.200600090

16. Taş NG, Gökmen V. Phenolic compounds in natural and roasted nuts and their skins: a brief review. Curr Opin in Food Sci. (2017) 14:1039. doi: 10.1016/j.cofs.2017.03.001

17. Coppin EA, Pike OA. Oil stability index correlated with sensory determination of oxidative stability in light-exposed soybean oil. J Am Oil Chem Soc. (2001) 78:13-8. doi: 10.1007/s11746-001-0212-4

18. Frankel EN. Lipid Oxidation. Cambridge: Elsevier Press (2014). p. 488.

19. Tinello F, Lante A, Bernardi M, Cappiello F, Galgano F, Caruso MC, et al. Comparison of OXITEST and RANCIMAT methods to evaluate the oxidative stability in frying oils. Eur Food Res Technol. (2018) 244:74755. doi: 10.1007/s00217-017-2995-y

20. Shahidi F, Zhong Y. Lipid oxidation and improving the oxidative stability. Chem Soc Rev. (2010) 39:4067-79. doi: 10.1039/b922183m

21. Li X, Wang SC. Shelf life of extra virgin olive oil and its prediction models. J Food Qual. (2018) 2018:1639260. doi: 10.1155/2018/1639260

22. Codex Alimentarius Commission. Codex Standard 33, Revised Codex Standard for Olive Oils, and Olive Pomace Oils, Standards and Standard Methods. Rome (2003). p. 4.

23. Makhoul H, Ghaddar T, Toufeili I. Identification of some rancidity measures at the end of the shelf life of sunflower oil. Eur J Lipid Sci Tech. (2006) 108:143-8. doi: 10.1002/ejlt.200500262

24. Idris NA, Abdullah A, Halim A. Evaluation of palm oil quality: correlating sensory with chemical analyses. J Am Oil Chem Soc. (1992) 69:2725. doi: 10.1007/BF02635900

25. Farhoosh R, Hoseini-Yazdi SZ. Shelf-life prediction of olive oils using empirical models developed at low and high temperatures. Food Chem. (2013) 141:557-65. doi: 10.1016/j.foodchem.2013.03.024 
26. Kochhar SP, Henry CJ. Oxidative stability and shelf-life evaluation of selected culinary oils. Int J Food Sci Nutr. (2009) 60(Suppl. 7):28996. doi: 10.1080/09637480903103774

27. Farhoosh R. Shelf-life prediction of edible fats and oils using Rancimat. Lipid Technol. (2007) 19:232-4. doi: 10.1002/lite.200700073

28. Gama T, Wallace HM, Trueman SJ, Hosseini-Bai S. Quality and shelf life of tree nuts: a review. Sci Hortic. (2018) 242:11626. doi: 10.1016/j.scienta.2018.07.036

29. Cämmerer B, Kroh LW. Shelf life of linseeds and peanuts in relation to roasting. LWT. (2009) 42:545-9. doi: 10.1016/j.lwt.2008.08.003

30. American Oil Chemists' Society. Official Methods and Recommended Practices of the AOCS. 7th ed. Champaign, IL: AOCS (2017).

31. Tuberoso CI, Kowalczyk A, Sarritzu E, Cabras P. Determination of antioxidant compounds and antioxidant activity in commercial oilseeds for food use. Food Chem. (2007), 103:1494501. doi: 10.1016/j.foodchem.2006.08.014

32. Boujemaa I, El Bernoussi S, Harhar H, Tabyaoui M. The influence of the species on the quality, chemical composition and antioxidant activity of pumpkin seed oil. OCL. (2020). 27:1-7. doi: 10.1051/ocl/2020031

33. Yang KM, Hsu FL, Chen CW, Hsu CL, Cheng MC. Quality characterization and oxidative stability of camellia seed oils produced with different roasting temperatures. J Oleo Sci. (2018) 67:389-96. doi: 10.5650/jos.ess 17190

34. Martínez ML, Penci MC, Marin MA, Ribotta PD, Maestri DM. Screw press extraction of almond (Prunus dulcis (Miller) DA Webb): oil recovery and oxidative stability. J Food Eng. (2013) 119:40-5. doi: 10.1016/j.jfoodeng.2013.05.010

35. Prescha A, Grajzer M, Dedyk M, Grajeta H. The antioxidant activity and oxidative stability of cold-pressed oils. J Am Oil Chem Soc. (2014) 91:1291301. doi: 10.1007/s11746-014-2479-1

36. Barroso AK, Pierucci AP, Freitas SP, Torres AG, Rocha-Leao MH. Oxidative stability and sensory evaluation of microencapsulated flaxseed oil. J Microencapsul. (2014) 31:193-201. doi: 10.3109/02652048.2013. 824514

37. Kasote DM, Badhe YS, Hegde MV. Effect of mechanical press oil extraction processing on quality of linseed oil. Ind Crops Prod. (2013) 42:103. doi: 10.1016/j.indcrop.2012.05.015

38. O'Keefe SF, Wiley VA, Knauft DA. Comparison of oxidative stability of high- and normal-oleic peanut oils. J Am Oil Chem Soc. (1993) 70:48992. doi: 10.1007/BF02542581

39. Miraliakbari H, Shahidi F. Lipid class compositions, tocopherols and sterols of tree nut oils extracted with different solvents. J Food Lipids. (2008) 15:8196. doi: 10.1111/j.1745-4522.2007.00104.x

40. Cao J, Deng L, Zhu X-M, Fan Y, Hu J-N, Li J, et al. Novel approach to evaluate the oxidation state of vegetable oils using characteristic oxidation indicators. J Agr Food Chem. (2014) 62:12545-52. doi: 10.1021/jf50 47656
41. Mengistie T, Alemu A, Mekonnen A. Comparison of physicochemical properties of edible vegetable oils commercially available in Bahir Dar, Ethiopia. Chem Int. (2018) 4:130-5. Available online at: https://reurl.cc/ 43Xblv

42. Codex Alimentarius Commission. Codex Standard 210, Revised Codex Standard for Named Vegetable Oils, Standards and Standard Methods. Rome (2009). p. 7.

43. Comandini P, Verardo V, Maiocchi P, Caboni MF. Accelerated oxidation: comparative study of a new reactor with oxidation stability instrument. Eur J Lipid Sci Technol. (2009) 111:933-40. doi: 10.1002/ejlt.200900009

44. Zhang CQ, Xu YJ, Lu YZ, Li LQ, Lan XZ, Zhong ZC. Study on the fatty acids, aromatic compounds and shelf life of Paeonia ludlowii kernel oil. J Oleo Sci. (2020) 69:1001-9. doi: 10.5650/jos.ess20084

45. Verardo V, Riciputi Y, Sorrenti G, Ornaghi P, Marangoni B, Caboni MF. Effect of nitrogen fertilisation rates on the content of fatty acids, sterols, tocopherols and phenolic compounds, and on the oxidative stability of walnuts. LWT. (2013) 50:732-8. doi: 10.1016/j.lwt.2012.07.018

46. Seppanen CM, Song Q, Saari Csallany A. The antioxidant functions of tocopherol and tocotrienol homologues in oils, fats, and food systems. J Am Oil Chem Soc. (2010) 87:469-81. doi: 10.1007/s11746-009-1526-9

47. Cao J, Li H, Xia X, Zou XG, Li J, Zhu XM, et al. Effect of fatty acid and tocopherol on oxidative stability of vegetable oils with limited air. Int J Food Prop. (2015) 18:808-20. doi: 10.1080/10942912.2013.864674

48. Zeng W, Endo Y. Lipid characteristics of camellia seed oil. J Oleo Sci. (2019) 68:649-58. doi: 10.5650/jos.ess18234

49. Nederal S, Škevin D, Kraljić K, Obranović M, Papeša S, Bataljaku A. Chemical composition and oxidative stability of roasted and cold pressed pumpkin seed oils. J Am Oil Chem Soc. (2012) 89:1763-70. doi: 10.1007/s11746-012-2076-0

Conflict of Interest: The authors declare that the research was conducted in the absence of any commercial or financial relationships that could be construed as a potential conflict of interest.

Publisher's Note: All claims expressed in this article are solely those of the authors and do not necessarily represent those of their affiliated organizations, or those of the publisher, the editors and the reviewers. Any product that may be evaluated in this article, or claim that may be made by its manufacturer, is not guaranteed or endorsed by the publisher.

Copyright (c) 2021 Tsao, Chang, Ho, Chuang and Lee. This is an open-access article distributed under the terms of the Creative Commons Attribution License (CC BY). The use, distribution or reproduction in other forums is permitted, provided the original author(s) and the copyright owner(s) are credited and that the original publication in this journal is cited, in accordance with accepted academic practice. No use, distribution or reproduction is permitted which does not comply with these terms. 